\title{
Chroniques d'un haute-contre en terres indiennes
}

Tales of a counter tenor in Indian lands

Jorge López Palacio

Traducteur : Sylvie Blasco

\section{OpenEdition}

\section{Journals}

Édition électronique

URL : http://journals.openedition.org/ethnomusicologie/1199

ISSN : 2235-7688

\section{Éditeur}

ADEM - Ateliers d'ethnomusicologie

\section{Édition imprimée}

Date de publication : 31 décembre 1995

Pagination : 147-158

ISBN : 2-8257-0537-3

ISSN : $1662-372 X$

\section{Référence électronique}

Jorge López Palacio, «Chroniques d'un haute-contre en terres indiennes », Cahiers d'ethnomusicologie [En ligne], 8| 1995, mis en ligne le 04 janvier 2012, consulté le 20 avril 2019. URL : http:// journals.openedition.org/ethnomusicologie/1199

Ce document a été généré automatiquement le 20 avril 2019

Tous droits réservés 


\section{Chroniques d'un haute-contre en terres indiennes ${ }^{1}$}

Tales of a counter tenor in Indian lands

Jorge López Palacio

Traduction : Sylvie Blasco

« Le chant est goajiro, la langue est goajira, mais

vous chantez comme un curé. »

Il me le dit les yeux dans les yeux et sans aucune pitié.

\section{La musique et le regard}


1 1971, mois de décembre. Péninsule de la Goajira colombienne, Nazareth. Notre panier s'ouvre. Nous en sortons une calebasse grande comme le ventre d'une femme enceinte et nous la déposons au centre de l'espace. Les enfants goajiro qui nous entourent, ouvrent de grands yeux : avec deux calebasses et un peu d'eau nous faisons un tambour. Sur une autre calebasse, nous plaçons un bâton au profil crénelé. Quand on le frotte avec une

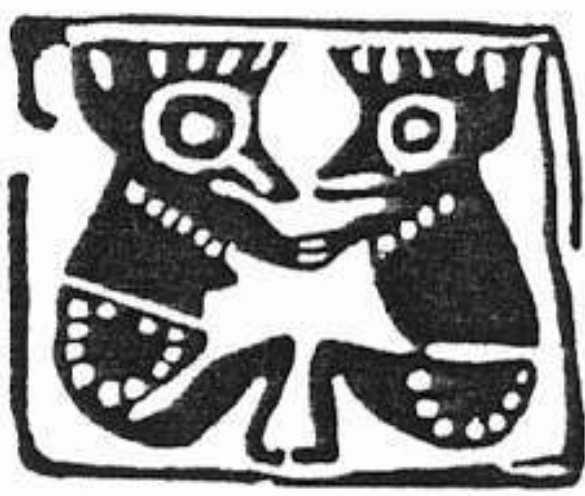
baguette, une voix de bête féroce sourd des entrailles de la terre. Les enfants s'accroupissent, appuient leurs coudes sur leurs genoux et reposent leurs petites têtes couleur de bronze, sur leurs mains. Alors arrivent les tambours sacrés des Aztèques, le teponaztli et le huehuetl, descendus du soleil par un escalier de carapaces de tortues. Les suit la maraka noire avec des points blancs, traces sans fin des fourmis. Les enfants se sont retournés : les adultes approchent. Nous sortons une flûte de Pan faite de tubes de carrizo ${ }^{2}$ et une flûte verticale d'os de cerf avec une embouchure de quena. Soudain, Firizai, la fille du soleil, sonnaille de graines d'une liane amazonienne, se met à chanter. Et voilà que l'arc de chasse prend place sur deux calebasses qui paraissent deux seins surgis de terre. Après lui viennent des pierres, de celles qu'ont tenues entre leurs mains les hommes du paléolithique colombien, il y a 14000 ans, et dont on peut faire de la musique en les frappant et en utilisant la bouche comme caisse de résonance. Leurs succèdent deux coquillages, tels deux grandes oreilles que l'on peut frotter ou percuter avec des coquilles d'huitres ou des cailloux. Les rejoignent les yapurutu, les flûtes géantes des Cubeo de la forêt amazonienne, et pour finir les kamu purrui, les flûtes de Pan des Cuna du Darién. Le panier vide sert de pupitre pour les livres de mythes et de légendes. Le projecteur de diapositives, dirigé vers un écran improvisé fait d'un drap tendu, est connecté au groupe électrogène du couvent tandis que les nonnes, palombes géantes, se tiennent à une prudente distance. Le concert commence sous un enchevêtrement de regards : regards d'enfants, regards de jeunes, d'hommes, de femmes, de vieux et regards de ces guerriers pur-sang qui viennent juste d'apparaître, armés de leurs arcs et de leurs flèches. Ils ont fait pacte de sang et juré de ne pas se « civiliser ». Première diapositive : une femme amazonienne nue et en couleur. Rires et commentaires. Un homme s'avance jusqu'à l'écran et touche la poitrine molle de la femme. Quelques jeunes étudient le mécanisme du projecteur. Les regards des enfants, rapides comme les colibris, vont d'instrument en instrument. Ceux, plus lents, des adultes nous observent. Les guerriers nous dévisagent sans ciller. L'un d'eux accroche mes yeux et un frisson parcourt ma colonne vertébrale. Personne ne m'a jamais considéré ainsi. Un regard sans interrogation, une paire de lances clouées dans mes yeux romantiques. Mes yeux fuient. Je me concentre sur notre concert-conférence pour les Indiens goajiro. J'argumente sur l'extrême richesse des cultures amérindiennes et sur leur nécessité de se connaître et de s'unir. Sur une carte du continent américain, je signale les lieux d'implantation des différents groupes. Au nord: les Inuit, les Ojibwa, les Navajo, les Apaches, les Sioux. Au Mexique : les Seri, les Yaqui, les Aztèques, les Maya. Au sud: les Cuna, les Kogi, les Bari, les Goajiro... Applaudissements et exclamations d'approbation... les Goahibo, les Piaroa, les Tukano, les Cubeo, les Bororo, les Quechua, les Aymara, les Ona... La musique avance tel un bateau plein d'images et de sons neufs. Diapositives, 
chants, lectures de mythes, fonctions des instruments musicaux. Quelques questions, la curiosité, l'étonnement lumineux des enfants, et la confiance s'installe peu à peu entre les adultes et nous. Les instruments, sculptures sonores, sont les lieux de convergence de nos différents points de vue. Nos regards se croisent, se tissent. Nous dansons et soufflons dans les kamu purrui, quand un jeune rompt la frontière de notre "espace scénique ", saisit un yapurutu et se met à jouer. Un autre lui emboîte le pas et s'empare d'un coquillage dont il sort un son rond qui va loin comme appelant quelqu'un. Un autre s'approche et un autre encore... Notre concert didactique s'écroule tel un château de cartes. Les guerriers pur-sang furent les seuls à garder la distance.

\section{L'ontoroyoi3 de Culebra Ipuana}

2 Le ciel de la Goajira est si pur que certaines étoiles peuvent y être vues pendant le jour. Quand je demande à Culebra Ipuana, comment dire étoile en sa langue, il me répond de sa voix forte, ferme, lancée vers le haut: jorotz! La terre de la Goajira est ocre, presque rouge, prolongée par ces organes des sens que sont les maisons. Maisons de chair, maisons vivantes. Quand je demande à Culebra Ipuana comment nommer, dans sa langue, la plante dont se servent les Goajiro pour construire leurs rancherías ${ }^{4}$, il me répond en accentuant fortement le troisième " 0 » : yotojoro! Nous avions rencontré Culebra dans un bar de la plage du Cabo de la Vela. Ce vieil et robuste Goajiro y buvait de l'alcool en compagnie d'autres hommes. J'avais alors sorti de notre panier un ontoroyoi que nous avions fabriqué à partir du dessin d'une revue d'anthropologie et j'avais joué les premières notes d'une improvisation dans le style goajiro. Surpris de voir un «arijuna », un étranger, jouer de leur instrument favori : le totoi, wotoroyoi ou ontoroyoi, les hommes s'étaient approchés de notre table. Sans me troubler, je continuai à improviser et Culebra, montrant l'instrument du doigt, m'apostropha : Nojotz! Un jeune homme bilingue, son fils, m'expliqua que l'instrument ne plaisait pas à son père et que celui-ci m'invitait à en connaître un véritable, chez lui à plus ou moins une heure de marche de là. 
Fig. 1 : Jorge López Palacio.

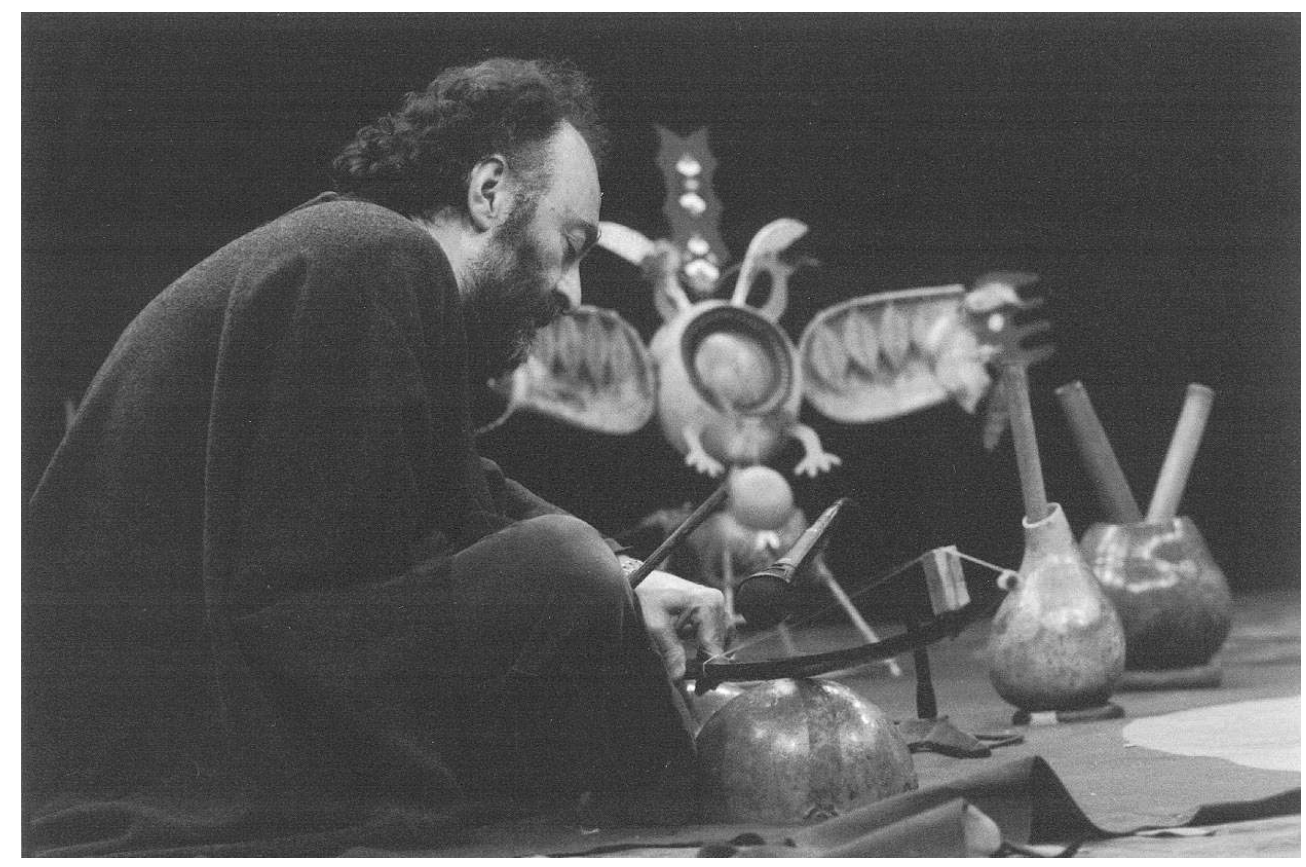

Photo : Isabelle Meister, Azzurro Matto

3 Nous laissons la mer Caraïbe derrière nous et, comme l'exige de nous Culebra, nous prenons le chemin de sa maison en file indienne, lui et son fils en tête, nous quatre au centre et les autres Goajiro fermant la marche. Tout en regardant de droite et de gauche comme craignant quelque chose, Culebra sort de sa poche une guimbarde de fer bien forgé et se met à jouer. Il joue bien. Nous arrivons au hameau, trois maisons faites de yotojoro, une espèce de cactus qui couvre de grandes extensions du désert et forme un labyrinthe de broussailles dans lequel les Goajiro se meuvent comme des poissons. Le vert du yotojoro tire vers le gris et s'ajoute au bleu du ciel, à la terre rouge, aux peaux chocolat. Les visages des femmes sont recouverts d'une peinture végétale les protégeant de la fureur du soleil et leurs mantas éclatent de couleurs. Culebra n'est pas riche mais il possède une histoire légendaire qu'il me chante, sa tête lourde de soixante années posée sur mon épaule. J'enregistre. Après chaque mélodie, il me fait réenrouler la bande et nous écoutons. Alors, pensif, avec un hochement de tête, il approuve : « C'est bien, elle ne ment pas ». Des heures passent. Les enfants suivent pas à pas Carlos Chávez, un musicien du groupe. Quand Carlos s'arrête, ils approchent leurs petites mains cuivrées de ses jambes et les caressent, fascinés par ce poil que les Indiens n'ont pas. Si Carlos bouge, les petites mains se détachent mais ne s'éloignent pas. Carlos s'arrête, elles reprennent leur promenade, des pieds déchaussés jusqu'au short. Les petites filles ont déjà leur manta de couleurs et l'une d'elles, de quelque deux ans et demi, se serre contre moi tandis que j'enregistre. Culebra, son père, voyant comme la petite m'apprécie, me dit : " Je vous la donne ». Je sursaute... C'est impossible! Culebra moitié furieux, moi plus effrayé encore... Son fils me traduit et m'explique que je ne peux refuser, j'appartiens déjà à la famille. Et moi, de répliquer que le froid de Bogota rend malade. Et moi, d'argumenter que la petite s'ennuiera loin de son papa, loin de sa maman, loin de ses petits frères. A force, Culebra se rend à mes objections et termine son chant, moitié ivre, délaissant la bouteille qu'il a vidée peu à peu comme buvant la vie. Carlos demande à prendre une photo et Culebra 
exige de poser de face, avec son arc et une flèche immense tendue vers l'objectif. Le bleu du ciel tourne au noir. Le fils de Culebra nous accompagne sur le chemin du retour. Dans notre panier, à côté de notre ontoroyoi de bambou des Andes, a pris place un merveilleux ontoroyoi «Stradivarius », que Culebra nous a offert et qui nous accompagnera durant de nombreuses années dans nos errances de troubadours.

\section{Messe de minuit}

4 Aremazaïn, messe de minuit. Dans l'église, des religieuses et un chœur de femmes indiennes chantent des villancicos ${ }^{5}$ espagnols en langue goajira. Sur le parvis, les hommes vont et viennent, boivent, discutent, exhibent leurs armes de tous calibres. Un Indien de haute taille passe devant moi en faisant tourner un pistolet automatique autour de son index et il m'adresse un sourire. Il porte un beau guayuco ${ }^{6}$ tissé par des mains d'artiste, des sandales, un chapeau et une chemise rouge. Il pénètre dans l'enceinte sacrée et se couche de tout son long sur un banc. Plus loin, un autre Goajiro cuve son vin en ronflant. Deux autres conversent avec animation, échangeant des bouteilles de whisky et d'eau de vie. Les villancicos se mêlent au vacarme des hommes. Un gros, grand et vieux Goajiro, et un autre maigre, petit et jeune, sortent enlacés, armés de leurs carabines automatiques. La messe suit son cours. Les femmes invectivent ces hommes qui ne savent rien respecter et le curé, encadré de ses enfants de chœur, accélère l'office, usant d'un latin vertigineux. Quand la petite cloche sonne le moment crucial de l'élévation, un cri et un coup de feu lui répondent. Alors des rires et des acclamations fusent et, dans un beau tollé, les Goajiro sortent fêter l'impertinent. Depuis le parvis, je vois le curé s'éloigner dans sa Toyota verte jusqu'à se perdre dans les ténèbres du désert.

\section{Une fenêtre}

La plage de sable blanc, la mer Caraïbe et le ciel sans fin. Nous descendons de notre Nissan Patrol pour respirer chacun de notre côté, cet air chargé de lumière. A nos pieds, les vagues déposent délicatement leur charge d'écume. Des bandes de vent arrivent du nord, d'autres croisent l'espace en provenance du sud, d'autres dévalent du ciel et toutes se mêlent en une tresse invisible. Une musique cristalline agite l'air. En pénétrant par la fenêtre ouverte de notre véhicule, le vent devient mélodie. Dans ce monde de sable, d'eau et de lumière, notre « tout terrain » se fit ocarina.

\section{Ici, il n'y a presque personne}

Dans la plus grande maison de Puerto Estrella, nous rencontrons une femme, belle, très grosse, avec un visage comme sculpté dans le bois. Elle tisse un hamac de couleurs vives sur un immense métier et, sans interrompre son travail, nous salue cordialement . Ses enfants, un garçon de 20 ans et une jeune fille de 17 ans, nous invitent à prendre un siège. Quand nous leur expliquons le but de notre visite, la réalisation d'un concert, ils nous répondent qu'ici, il n'y a presque personne, seulement eux, l'autre et le vent. Lui porte un diadème tissé en fibres végétales et de son front sortent trois plumes de paon. Elle, est chaussée de sandales qu'ornent de gros pompons couleur carotte. A ses mains, je découvre une bague ${ }^{7}$ en or où l'on peut lire gravé autour d'une émeraude: 1970 - 
Promotion Ernesto Che Guevara. Collège Simon Bolivar. Maracaibo. Vénézuela. Quand je lui rends la bague, elle sort en courant poursuivre une poule noire, un balai à la main. Au même moment entre un homme d'une trentaine d'années. Il s'appelle Juan. Il nous confirme qu'ici il n'y a presque personne, seulement eux et le vent, et nous invite à découvrir les récifs et la furie de la mer. Tandis que nous approchons du vacarme des vagues, apparaissent à fleur de sable, des os, des crânes et des planches, restes de croix. La flûte de Pan que je porte à la main chante seule de recevoir en ses orifices de bambou, les serpents de vent de l'océan. Juan s'essaie à la jouer mais sa respiration ne peut rien contre celle de la mer. Alors, parmi les os presque fossiles et devant l'apocalypse des vagues, je donne à Juan sa première leçon de flûte. En revenant au village, il me demande de lui laisser ce bel instrument qu'il me promet de garder avec soin et de bien jouer. Je le lui donne sans regret, car si ici il n'y a presque personne, il y a pour le moins, Juan, quelques autres et le vent.

Fig. 2a : Quelques instruments amérindiens de Jorge López Palacio.

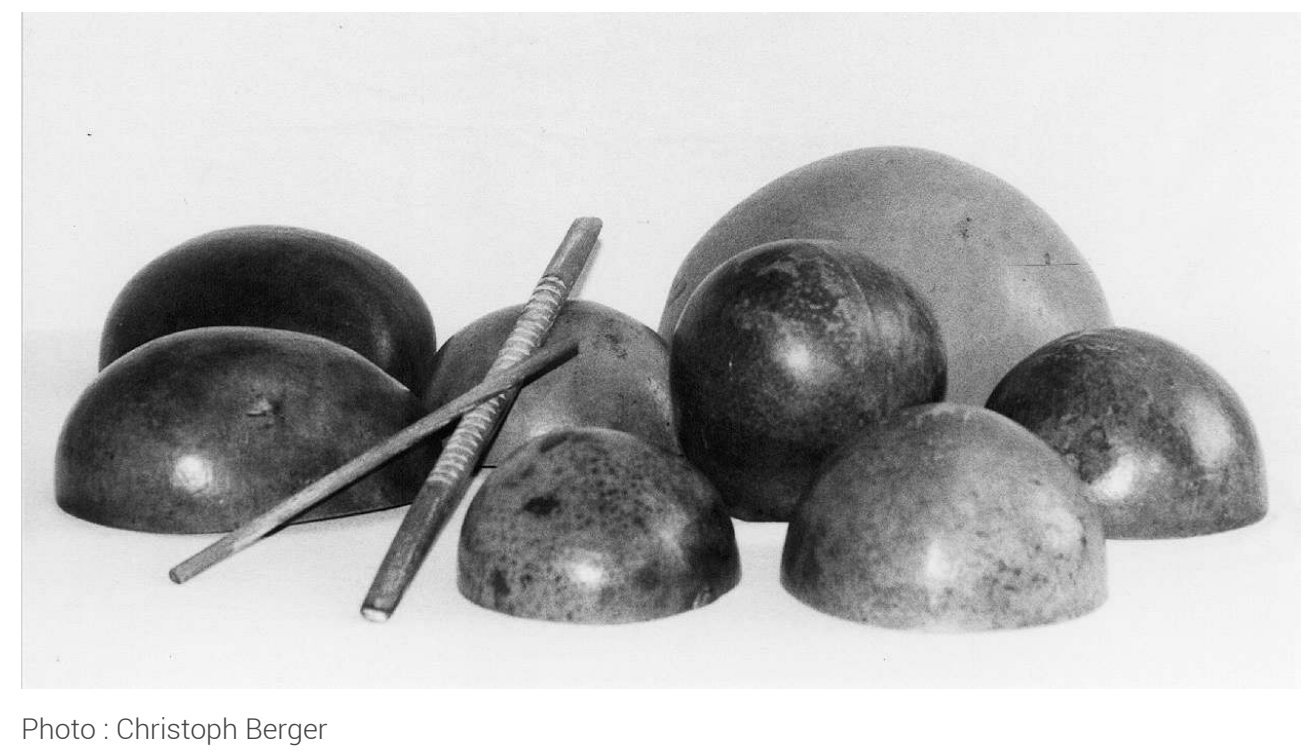

\section{Réinventer la musique}

7 Le chemin est tortueux, bordé de plantations de bananiers, de canne à sucre et de maïs. Nous avançons lentement. Les fleurs sauvages semblent nous crier des insultes de fleurs offensées par notre présence motorisée. Quand le camion s'enfonce dans la boue, les Indiens se précipitent et avec leurs bras, le camion repart. Voilà le village. Les gens y sont déjà rassemblés. Le concert commence quand le chef de la communauté, un homme d'une soixantaine d'années, se plante devant nous et dit avec émotion : "Compañeros, c'est une bien grande peine, mais nous avons perdu notre musique, nous avons perdu notre langue et toutes nos coutumes. Il ne nous reste plus que notre nom, $\mathrm{Pijao}^{8}$, et notre misère. Comment récupérer notre musique?» - «Compañero, si la musique est perdue, il faut la réinventer ». Il demande de nouveau: "Comment la réinventer? Nous n'avons pas d'instruments et nous ne savons pas à quoi cette musique ressemblait.» - «Eh bien! Choisissez quelques instruments parmi ceux que voici, organisez un groupe et la musique, qu'elle ressemble ou non à la vôtre d'il y a cinq siècles, viendra seule. » 
De tous les instruments, il ne choisit que quelques flûtes traversières. Le savait-il ? C'étaient justement les flûtes de ses voisins et ennemis ancestraux, les Paez. Un an plus tard nous arriva la bonne nouvelle qu'un groupe de musiciens Pijao avait participé à une rencontre culturelle intercommunautaire. Nous ne sûmes jamais rien ni sur les rythmes ni sur les mélodies, mais ce que nous comprîmes, c'est que les flûtes sont des êtres vivants, qu'elles portent une semence.

Fig. 2b : Quelques instruments amérindiens de Jorge López Palacio.

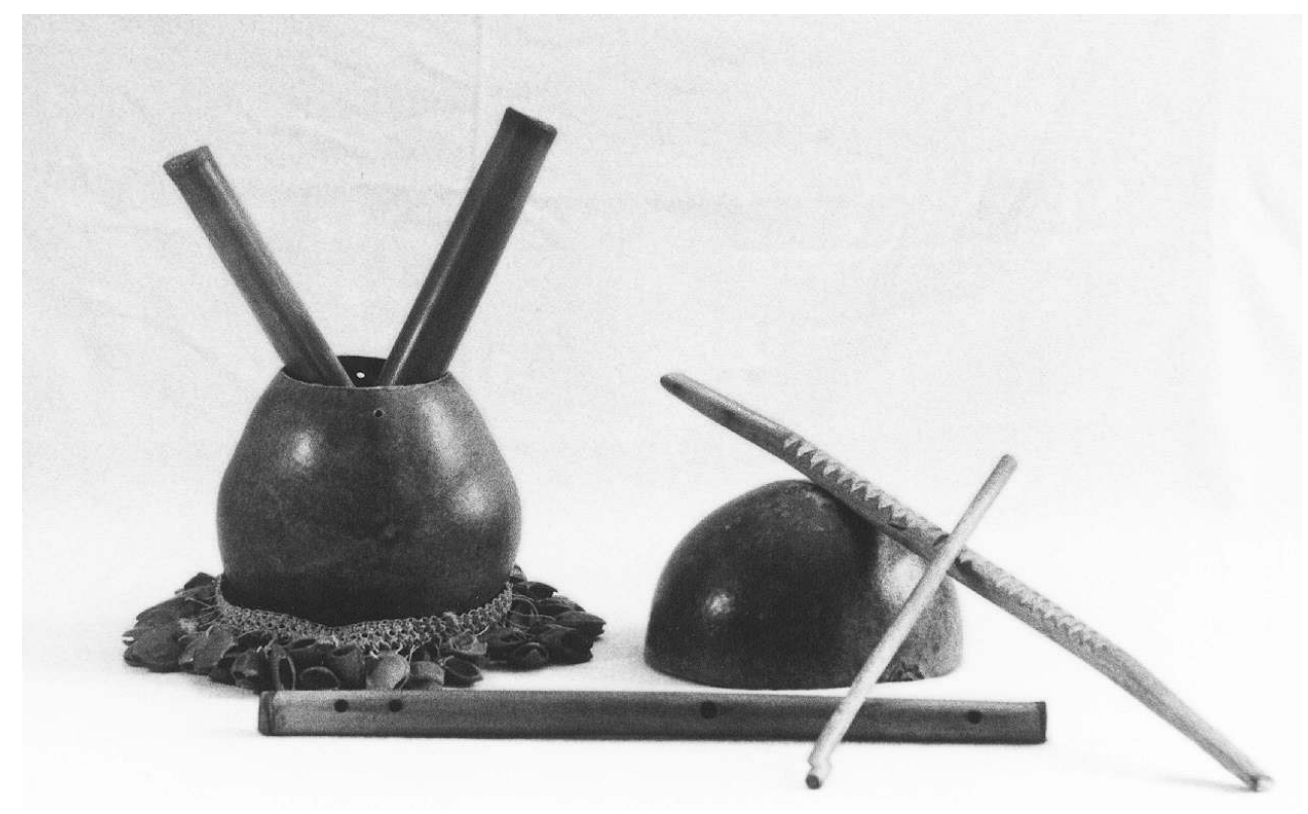

Photo : Christoph Berger

\section{Yoino}

Nous chargeons les chevaux et amorçons la descente depuis le paramo ${ }^{9}$. De là-haut, le soleil semblait naître de par le bas, comme s'il émergeait d'une blessure de la terre. Peu à peu les frailejones ${ }^{10}$, ces plantes aux feuilles telles des oreilles de lapin, disparaissent et nous découvrons un paysage de cordillères vertes et bleues taillées au couteau dans le versant oriental de la chaîne centrale des Andes du Cauca. Les instruments dorment dans leurs caisses rouges et les chevaux avancent en mesurant les abîmes de leurs yeux animaux qui voient sans regarder. Nos pieds ignorants des chemins autres que ceux simples des trottoirs, trébuchent. A nos côtés, les Indiens Paez qui dirigent l'expédition, courent, sautent de pierre en pierre, avec une sûreté féline. Voilà le premier village : dix humbles maisons sur la crête d'une cordillère qui se détache d'une autre plus grande, comme une côte de la colonne vertébrale. Une fois de plus nous étendons nos tapis et plaçons nos instruments au centre de la petite place publique. Une fois de plus notre concert voyage du nord au sud, ponctué d'explications sur l'origine et les fonctions des instruments ou, occasionnellement, de la narration de mythes et de légendes appris durant nos visites à d'autres groupes ou extraits de livres d'anthropologie. Aujourd'hui, je raconte le mythe de création des Indiens Kogi de la Sierra Nevada de Santa Marta. «Au commencement, il n'y avait ni la terre, ni les animaux, ni les gens, ni les plantes, seulement Naowa, l'eau, la mère. La mère donna naissance à un premier fils, Bunkua Nabi. 
Il vivait dans l'eau, dans une maison faite d'écume. Il se sentit bientôt seul. «Mère, dit-il, donne-moi des frères ». Sa mère lui donna des frères: Sintana, Bunkua Tse, Noana Tse, Seyankua, Kimaku, Kakarabiku. Alors ceux-ci demandèrent à leur mère : "Mère, donnenous de la terre pour semer ». La mère leur donna la première de ses neuf filles, «Sable de la plage ». Ils voulurent semer, mais rien ne poussa. Le maïs ne sortit pas, la graine mourut. Ils demandèrent à leur mère une nouvelle terre et elle leur donna "Argile rouge ». Ils semèrent, mais là encore, rien ne vint. Une troisième fois, ils demandèrent. La mère leur donna une terre plus sombre mélée de pierres et de sable. De là, sortit une frêle plante, mais elle n'eut pas de fruit. Ils demandèrent de nouveau... de nouveau encore... Bientôt il ne resta plus que la neuvième terre, "Terre noire ", la fille préférée de Naowa. Ils la demandèrent. Rien. La mère refusa et elle cacha sa fille dans une chambre. Alors ses fils coupèrent deux tubes de carrizo et, avec de la cire d'abeille et deux tuyaux de plumes, ils fabriquèrent deux flûtes : l'une, la femelle avec cinq trous, l'autre, le mâle, avec deux trous. Au mâle, ils donnèrent une compagne, la petite maraka. Avec ces flûtes, ils jouèrent durant des jours et des nuits, dansant autour de la chambre dans laquelle était enfermée Terre noire, la mère des cultures. Bientôt Terre noire n'y tint plus, elle glissa sous la porte et s'enfuit à la suite des musiciens. Alors, en virevoltant et en dansant, ils recouvrirent les pentes de la Sierra de terre noire, et les arbres poussèrent et les animaux et les gens peuplèrent la forêt. »

Yoino, un chamane m'écoute avec attention. Quand je termine, il se lève et prend la parole : «C'est bien. Maintenant je vais vous dire comment nous sommes nés, nous les Paez. Ensuite vous irez à la Sierra Nevada et vous leur raconterez. En haut, dans le paramo, il y a un petit lac très profond. Personne n'en a jamais touché le fond. La nuit, on y entend le tonnerre résonner. C'est Juantama, notre plus ancien cacique. Juantama a un corps de lézard, des écailles vertes et des yeux rouges, immenses, qui voient tout. De sa bouche et de son nez, coulent sans jamais s'interrompre tant et tant de bave et de morve que sa poitrine est en permanence recouverte de mouches et de vers. Juantama possède un bâton de commandement en or avec lequel il s'amuse à frapper les pierres, là-haut dans le petit lac du paramo. Chaque coup de tonnerre est un coup de bâton. Il y a de nombreuses années, le président de la République colombienne, invita Juantama à venir le voir dans son palais de Santa Fé de Bogotá. Juantama monta sur son tapis volant, traversa la cordillère des Andes, survola les plaines de la Magdalena et atterrit sur la place Bolivar de la capitale, là où l'attendait le président et ses ministres. Juantama s'approcha et le président sortit de la poche de son smoking un fin mouchoir blanc brodé de fils d'argent avec lequel il entreprit le nettoyage de la bave et de la morve. Juantama, reconnaissant, lui offrit un bâton, plus petit que le sien, mais suffisamment puissant pour que le président puisse s'en divertir en gouvernant et en provoquant des tempêtes. Ce que nous disons, c'est que nous les Paez, venons des coups de bâton de Juantama contre les rochers du lac.» Trois nuages en forme d'œufs se placèrent devant le soleil et celui-ci dut faire mille acrobaties pour continuer à être vu. Alors, nous reprîmes notre descente, laissant derrière nous un sillage de chants, de mythes et d'affection.

\section{Après la tempête, le calme}

11 «Compañero, pourquoi dire que des petits blancs qui jouent la musique indienne, ça ne vous plaît pas? Compañero, vous n'avez pas le droit de nous interdire de jouer cette musique. Vous êtes un vénérable ancien, vous êtes un grand dirigeant indien mais vous 
êtes injuste. Vous ignorez nos efforts pour faire découvrir cette merveilleuse musique. Vous ignorez nos efforts pour que les Indiens ne soient plus considérés comme des animaux et pour que leur culture, riche en art, en pensée, en esprit créateur soit reconnue comme faisant partie du patrimoine de l'humanité. Et enfin, compañero, je n'ai jamais demandé d'autorisation à personne pour chanter." Quelques étudiants de l'Université de Caldas à Manizales, ville à cheval sur la cordillère des Andes, m'applaudirent. D'autres me rappelèrent que je devais respecter le compañero, car c'était un vrai Indien. Nous nous retirâmes furieux de l'assemblée organisée par le comité de solidarité avec les luttes indiennes. Plus tard, Marcos Avirama, dirigeant du conseil indien de la région du Cauca, nous invita à une réunion avec le comité exécutif de son organisation. Il nous présenta ses excuses pour l'attitude désobligeante du compañero et manifesta un notable intérêt pour notre labeur de diffusion et de défense des cultures indiennes. Le vénérable ancien me regardait avec un mélange de rage et de sympathie et à la sortie, il me dit en approchant sa voix de terre de mon oreille : «C'est bon, faites-le, mais faites-le bien! » Et nous prîmes congé avec une accolade que jamais je n'oublierai.

\section{Mourir sur le ciment frais aux côtés de deux cadavres de cerfs}

12 Je suis là, planté sur le trottoir. J'attends l'avocat du travail de la province du Vichada, à Puerto Carreño, en Colombie, sur les bords de l'Orénoque. Je suis coordinateur de l'enseignement au secrétariat de l'éducation. Je suis là, appuyé au mur. J'attends l'avocat du travail qui ne viendra pas car il est ivre dans le bordel du port. Je l'attends pour l'informer, pour la énième fois, que je ne suis pas inscrit sur la liste des salariés bien que je sois nommé et que je sois déjà en train d'accomplir mes fonctions avec le plus grand sérieux. Je suis là, avec mes angoisses, quand apparaît au coin de la rue, en file indienne, un groupe de chasseurs goahibo armés d'arcs et de flèches et portant sur leurs épaules deux cerfs morts. Ils déposent les cerfs à mes pieds. Je m'incline, les caresse, et dans leurs yeux, la mort me regarde.

13 Les Goahibo ont déjà saisi leurs outils. Depuis plusieurs semaines, ils œuvrent au revêtement de la rue, embauchés par le secrétaire des travaux publics, Ignacio Puentes, qui vient à peine d'arriver, en retard, furibond au sortir d'une nuit blanche. Un Goahibo met en marche la bétonneuse; d'autres mélangent l'eau, le sable et la pierre; d'autres transportent dans leurs brouettes de fer, le ciment prêt à l'emploi. Ils sont ainsi occupés quand apparaît, à l'angle de la maison paroissiale, «le Corbeau », le très craint exguérillero libéral. Il conduit sa lente et vieille Harley Davidson, celle-là même qu'il échangea au Vénézuela, à un contrebandier yankee contre son merveilleux cheval blanc "Pigeon ». Comme toujours, il conduit en lisant le journal et chacun sait qu'il faudra se garer, car ne dit-on pas que «le Corbeau » est un homme «très dangereux »? Sur la surface fraîche de la rue, "le Corbeau » avance sans se troubler, laissant derrière lui, l'empreinte impeccable de sa Harley Davidson. Alors l'ingénieur Puentes saisit une brouette pleine de sable et la jette contre les roues de la moto. "Le Corbeau» saute, retombe sur ses deux pieds. L'ingénieur se lance à l'attaque, une machette à la main, «le Corbeau » sort un revolver noir, étend le bras et avec une surprenante sérénité, laisse partir le coup. Les Goahibo et moi regardons sans bouger. La détonation frappe contre le ciel, redescend et rebondit plusieurs fois contre la terre. Quand l'ingénieur reçoit la balle au milieu du front, il ne perd pas l'équilibre, il ne s'évanouit pas. Lentement, l'ingénieur 
s'asseoit. Puis il s'allonge et comme mesurant le temps, dépose délicatement sa machette sur sa poitrine. Ainsi prêt, il entre dans une agonie de trois jours et trois nuits. Maintenant Jaramillo, le policier, passe peureusement la tête et voyant « le Corbeau » son revolver à la main, se retire précipitamment. «Le Corbeau » lui crie : «Jaramillo, n'ayez pas peur, venez! Je vous remets mon arme et je vous accompagne au poste. Venez, Jaramillo, je ne vous ferai aucun mal! Jaramillo s'approche en tremblant et reçoit, reconnaissant, le revolver. «Le Corbeau » lui prend le bras et ensemble ils disparaissent par l'angle droit de la rue.

14 La nuit, dans leur campement, les Goahibo m'invitèrent à chanter et à danser et, selon ce que m'expliqua l'un d'eux, les témoins de la tragédie contaient dans leurs chants, les détails du douloureux événement.

\section{Un masque vivant}

15 Je me souviens de ce concert à Leticia, port colombien sur l'Amazone. Un public d'Indiens, de métis et de blancs. La police nous entoure, noyée dans le bruit d'incendie des insectes. De tous côtés les lucioles, ces minuscules étoiles ailées. Un air d'eau. Nous chantons un chant inuit, quand arrive un camion de police, transportant un groupe d'Indiens tikuna, hommes, femmes et enfants. Les policiers leur ordonnent de monter sur scène. Précipitamment nous rangeons nos instruments. Déjà, les Indiens envahissent l'espace et continuent un rituel qu'ils ont débuté quelques heures auparavant dans leur campement. Les femmes chantent et dansent, chacune traçant sur le sol avec un bâton de bois, une ligne imaginaire. Face à elles et avec un même rythme régulier, les hommes chantent et dansent clouant verticalement sur la ligne imaginaire un autre bâton. Soudain, monte sur scène un homme vêtu d'un masque qui lui couvre tout le corps, un masque fait de yanchama (toile d'écorce) et dont la tête est taillée dans le cœur du balsa. Un immense pénis de bois pousse tel un pistil depuis une paire de testicules faits de petites calebasses. Cet insolite personnage masturbe son impressionnant pénis en le dirigeant vers le public. Il copule avec les lignes imaginaires tracées sur le sol, il jette son sperme mythique de droite, de gauche, vers le ciel, il féconde les étoiles, les microphones, les haut-parleurs... Et le sperme mythique atteint le premier rang du public. Là, les filles des notables se couvrent le visage de honte. Alors retentissent des ordres de sortir ces Indiens sales car ici il y a des dames et, tandis que le public se partage entre ceux qui rient aux éclats et ceux qui maudissent ces Indiens du démon, les Tikuna sont réembarqués dans le camion de la police. Le jour suivant nous prîmes notre avion, regrettant de ne pas les avoir rencontrés.

\section{NOTES}

1. Traduit de l'espagnol par Sylvie Blasco

2. carrizo : variété de bambou

3. ontoroyoi : sorte de clarinette goajiro 
4. rancherías : groupements de maisons

5. villancico : chant de Noël

6. guayuco : pagne

7. En Amérique latine, chaque élève ayant obtenu le baccalauréat se fait faire une bague en souvenir, bague dont le modèle et l'inscription sont choisis par toute la promotion.

8. Pijao : groupe indien de la vallée et de la cordillère centrale des départements du Huila et du Tolima en Colombie.

9. paramo: lieu d'altitude élevée et très froid dans les Andes colombiennes, caractérisé par une végétation surtout constituée de frailejones.

10. frailejon: (Speletia grandiflora) plante à feuilles velues qui pousse sur les sommets et les pentes des paramo et donne une fleur jaune ressemblant au tournesol.

\section{RÉSUMÉS}

Il s'agit d'un voyage à l'intérieur d'un labyrinthe qui commence dans le « sujet » et se prolonge dans «l'objet », ces personnages jumeaux du mythe et du drame de la connaissance occidentale. Il s'agit d'un authentique voyage qui vise la connaissance et utilise une méthode de recherche « impure », le concert. Le sujet qui cherche s'expose sur la scène et se transforme en objet. Dans le désert de la Goajira colombienne, un jeune haute-contre et anthropologue libère sa voix d'ange. Un indien goajiro lui donne alors sa première leçon d'ethnomusicologie vivante : «Le chant est goajiro, la langue est goajira, mais vous chantez comme un curé ». Sur une plage, parmi les ossements humains qui jonchent le sable, le haute-contre donne à Juan, un autre indien goajiro, sa première leçon de flûte de Pan andine. Un ontoroyoi, clarinette goajira, fait fonction de prisme, prisme d'où surgissent les multiples lignes de contact possibles entre la famille de Culebra Ipuana et le chercheur. Dans l'immensité du désert, la Nissan Patrol des chercheurs devient instrument de musique. A Leticia, port colombien sur le fleuve Amazone, se rejoignent la danse phallique d'un chamane tikuna et un camion de la police. A Puerto Carreño, sur les bords de l'Orénoque, des chasseurs goahibo se transforment en ouvriers tandis que meurt un ingénieur. Dialectique et dynamique des regards lors d'un concert-conférence. Les villancicos et les armes à feu. Un peuple et les flûtes de ses ennemis. Un sillage de chants, de mythes et d'affection. Tel est le kaléidoscope de « l'objectivité » proposé par le haute-contre Jorge López Palacio.

It concerns a journey inside a labyrinth that starts with the 'subject' and extends to the 'object', twin characters of myth and drama in terms of western knowledge. It concerns a real journey that aims at knowledge and uses an impure means of research, the concert. The subject that searches stands on stage and is transformed into an object. In the Colombian Goajira desert, a counter tenor anthropologist lets out his angelic voice. A Goajiro Indian gives him his first living lesson in ethnomusicology : «Goajiro is the song, Goajiro is the language, but you, you sing like a priest. " On a beach, strewn with human bone remains the counter tenor gives Juan, another Goajira Indian his first flute lesson on the Andean Panpipes. A Goajiran clarinet, the ontoroyoi, acts as a prism through which appear the possible means of contact between the Culebra Ipuana family and the researcher. In the desert vastness the researcher's Nissan Patrol becomes a musical instrument. In the Colombian port of Leticia on the Amazon river, a Tikauna shaman's phallic dance encounters a police vehicle. In Puerto Carreño, on the banks of the river Orenoque, Goahibo hunters turn into workers whilst an engineer meets his death. During a concert- 
demonstration session there is a dialectic and dynamic of viewpoints. The villancicos and firearms. A people and the flutes of its enemy. A wake of songs, myths and affection. Such is the kaleidoscope of « objectivity » put forward by the counter tenor Jorge López Palacio.

\section{AUTEURS}

\section{JORGE LÓPEZ PALACIO}

Jorge López Palacio, chanteur et anthropologue colombien, est l'un des acteurs et des promoteurs latino-américains du dialogue entre les cultures du monde. En 1970, il créa à l'Université Nationale de Colombie, le groupe Yaki Kandru afin d'étudier et de diffuser la musique et les cultures indigènes du continent américain et de contribuer ainsi au rétablissement de la communication qui, comme l'écrit Claude Lévi-Strauss dans « Tristes Tropiques ", existait avant la conquête entre les différentes populations indigènes. De sa fondation à nos jours, ce groupe réalisa plusieurs disques et des centaines de concerts en Amérique Latine, en Europe et en Afrique. Du travail pédagogique basé sur la solidarité avec les communautés indiennes, Jorge López Palacio a reçu les enseignements musicaux, philosophiques et humains qui orientent sa réflexion anthropologique. Durant dix ans, il fût professeur des techniques de la voix à l'Ecole Nationale d'Art Dramatique de Bogotá. Résident en France depuis 1982, il enseigne l'anthropologie de la voix au Centre International de Musicothérapie à Paris ainsi qu'au Centre Feldenkrais - Cristoph Berger à Bâle et collabore aux Ateliers d'ethnomusicologie de Genève. 